\title{
THE $p$-CLASSES OF A HILBERT MODULE
}

\author{
JAMES F. SMITH
}

\begin{abstract}
Let $H$ be a right Hilbert module over a proper $H^{*}$-algebra $A$. For $0<p \leqq \infty$, an extended-real value $\|f\|_{D}$ is associated with each $f \in H$, and the $p$-class $H_{p}$ is defined to be $\left\{f \in H:\|f\|_{n}<\infty\right\}$. For $1 \leqq p \leqq \infty,\left(H_{p},\|\cdot\|_{p}\right)$ is a right normed $A$-module. If $1 \leqq p \leqq 2$, there is a conjugate-linear isometry of $\left(H_{p},\|\cdot\|_{p}\right)$ onto the dual of $\left(H_{q},\|\cdot\|_{q}\right)$, where $(1 / p)+(1 / q)=1$; hence $H_{p}$ is complete in its norm.
\end{abstract}

1. Introduction. Let $A$ be a proper $H^{*}$-algebra with inner product and norm denoted by $\langle\cdot, \cdot\rangle$ and $|\cdot|$, respectively. By a projection in $A$ we mean a nonzero selfadjoint idempotent, and by a projection base for $A$ we mean a maximal family of mutually orthogonal projections. The trace class of $A$, denoted by $\tau A$, is the set $\{x y: x, y \in A\}$. It is shown in [6] that a trace functional $\operatorname{tr}$ is unambiguously defined on $\tau A$ by letting $\operatorname{tr} x y=\left\langle x, y^{*}\right\rangle=$ $\sum\left\langle x y p_{\omega}, p_{\omega}\right\rangle$, where $\left\{p_{\omega}: \omega \in \Omega\right\}$ is any projection base for $A$. It is further shown that for each nonzero $a \in A$ there exists a unique positive element $[a] \in A$ (that is, one possessing the property $\langle[a] x, x\rangle \geqq 0$ for every $x \in A$ ) such that $[a]^{2}=a^{*} a$; moreover, $a \in \tau A$ if and only if $[a] \in \tau A$. A norm $\tau$ is defined on $\tau A$ by letting $\tau(a)=\operatorname{tr}[a]$; then $(\tau A, \tau)$ is a Banach *-algebra ([6], [5]). In [7] the present author has shown that each nonzero positive element $b$ of $A$ has a unique spectral representation $b=\sum \lambda_{n} e_{n}$, where the $\lambda_{i}$ are positive numbers with $\lambda_{i}<\lambda_{j}$ if $i>j$, and the $e_{i}$ are mutually orthogonal projections. In particular, for any nonzero $a \in A$, if $\sum \lambda_{n} e_{n}$ is the spectral representation of $[a]$, we define $|a|_{p}$, for $0<p<\infty$, by $|a|_{p}^{p}=$ $\sum \lambda_{n}^{p}\left|e_{n}\right|^{2}$. We also define $|a|_{\infty}$ to be $\lambda_{1}$, and $|0|_{p}=0$ for $0<p \leqq \infty$. The $p$-class $A_{p}, 0<p \leqq \infty$, is then defined as $\left\{a \in A:|a|_{p}<\infty\right\}$. Among the results of [7] are the following: (1) $|a|_{\infty}=\left\|L_{a}\right\|$, where $L_{a}$ denotes, as usual, the left multiplication operator; (2) $A_{p} \subset A_{p^{\prime}}$, if $0<p<p^{\prime} \leqq 2$, the inclusion being proper if $A$ is infinite-dimensional; and $A_{p}=A$ if $p \geqq 2$; (3) $\left(A_{2},|\cdot|_{2}\right)=(A,|\cdot|)$ and $\left(A_{1},|\cdot|_{1}\right)=(\tau A, \tau) ;$ (4) $\left(A_{p},|\cdot|_{p}\right)$ is a normed $*$-algebra for $1 \leqq p \leqq \infty$, and is complete for $1 \leqq p \leqq 2$.

A (right) Hilbert $A$-module $H$, introduced by Saworotnow in [4], is a complex linear space which is a right module over the proper $H^{*}$-algebra

Presented to the Society, March 27, 1972; received by the editors February 3, 1972. AMS (MOS) subject classifications (1970). Primary 46H25; Secondary 46K15, $46 \mathrm{C} 10$.

Key words and phrases. Hilbert module, $H^{*}$-algebra, trace class, $p$-classes, normed module.

(c) American Mathematical Society 1973 
$A$, and on which there is defined a vector inner product $(\cdot, \cdot)$ mapping $H \times H$ into $\tau A$, such that for elements of $H:(1)(f+g, h)=(f, h)+(g, h)$; (2) $(f, g)=(g, f)^{*} ;(3)(f, g a)=(f, g) a$, where $a \in A ;(4)(f, \alpha g)=\alpha(f, g)$ for any complex number $\alpha$; (5) if $f \neq 0$ then $(f, f)=a^{2}$ for some (unique) positive element $a \neq 0$ in $A$; we denote this $a$ by $[f] ;(6) H$ is complete in the norm $\|\cdot\|$ derived from the inner product $[\cdot, \cdot]$ defined by $[f, g]=\operatorname{tr}(g, f)$. Basic properties of Hilbert $A$-modules are obtained in [4], among them the fact that $\|f a\| \leqq\|f\||a|$ for $f \in H, a \in A$; hence a Hilbert $A$-module is evidently a particular instance of a Banach module (see [2, p. 263]). (We shall assume without loss of generality that $H$ is a faithful module, since its right annihilator $R$ is a closed two-sided ideal of $A$; hence $H$ is atways a faithful Hilbert $R^{\perp}$-module.) Giellis [1] has defined the trace class of $H$ to be $\tau H=\{f a: f \in H, a \in A\}$ and has defined a norm $\pi$ on $\tau H$ by $\pi(f)=$ $\tau([f])$. He has shown that $(\tau H, \pi)$ is a Banach module and has presented results on duality relationships.

Our present aim is to generalize the results of [1] for Hilbert modules, much as those of [5] and [6] were generalized for $H^{*}$-algebras in [7]. For $f \in H$, and for $0<p \leqq \infty$, we define $\|f\|_{p}=|[f]|_{p}$, and we let $H_{p}=$ $\left\{f \in H:\|f\|_{p}<\infty\right\}$ (that is, $f \in H_{p}$ if and only if $[f] \in A_{p}$ ). Our results are the following.

THEOREM 1. For $0<p<p^{\prime} \leqq \infty$ and any $f \in H,\|f\|_{p^{\prime}} \leqq\|f\|_{p}$; hence $H_{p} \subset$ $H_{p^{\prime}}$, and $H_{p}=H$ if $p \geqq 2$. For $1 \leqq p \leqq \infty,\left(H_{p},\|\cdot\|_{p}\right)$ is a right normed $A$ module. $\left(H_{2},\|\cdot\|_{2}\right)=(H,\|\cdot\|)$, and $\left(H_{1},\|\cdot\|_{1}\right)=(\tau H, \pi)$.

THEOREM 2. For $1 \leqq p \leqq 2$, let $q$ be such that $(1 / p)+(1 / q)=1$. Then there exists a conjugate-linear isometry of $\left(H_{p},\|\cdot\|_{p}\right)$ onto the dual of $\left(H_{q},\|\cdot\|_{q}\right)$; hence $H_{p}$ is complete in its norm.

We conclude with a necessary and sufficient condition for the inclusion $H_{p} \subset H_{p^{\prime}}$ to be proper $\left(1 \leqq p<p^{\prime} \leqq 2\right)$.

2. Preliminary results. We recall first of all from [4, Lemma 1] that $(f a, g)=a^{*}(f, g)$ for any $f, g \in H$ and $a \in A$. From the fact that $\tau(f, g) \leqq$ $\|f\|\|g\|[4$, Theorem 2], it is easily established that $(\cdot, \cdot)$ is $\tau$-continuous on $H \times H$ and therefore $|\cdot|$-continuous as well, since $\tau$ dominates $|\cdot|$ [6, Corollary 3]. We observe that $[f, g a]=\operatorname{tr}(g a, f)=\operatorname{tr} a^{*}(g, f)=$ $\langle(g, f), a\rangle$; similarly, $[f a, g]=\langle a,(f, g)\rangle$. Also, $[f, g a]=\operatorname{tr}(g, f) a^{*}=$ $\operatorname{tr}\left(g, f a^{*}\right)=\left[f a^{*}, g\right]$.

As in [1], we define the following sets of bounded linear transformations:

$$
\begin{aligned}
R(A) & =\{T: A \rightarrow A \mid T(a b)=(T a) b \text { for all } a, b \in A\}, \\
R(A H) & =\{T: A \rightarrow H \mid T(a b)=(T a) b \text { for all } a, b \in A\}, \\
R(H A) & =\{T: H \rightarrow A \mid T(f a)=(T f) a \text { for all } f \in H, a \in A\} .
\end{aligned}
$$


Still following [1], we define $L_{f} \in R(H A)$ for each $f \in H$ by $L_{f} g=(f, g)$. (Note that, by standard notation, $L_{[f]}$ is the operator effecting left multiplication by $[f]$ in $A$; clearly, $L_{[f]} \in R(A)$.) For $f \in H$, we shall define $T_{f} \in R(A H)$ by $T_{f} a=f a$. By a remark of Giellis $\left[1\right.$, p. 65], we have $T_{f}^{*} T_{f}=$ $L_{[f]}^{2}=L_{[f]^{2}}$. The relationship $T_{f}=L_{f}^{*}$ also holds, since for any $f, g \in H$ and $a \in A,\left[g, T_{f} a\right]=[g, f a]=\langle(f, g), a\rangle=\left\langle L_{f} g, a\right\rangle=\left[g, L_{f}^{*} a\right]$.

Lemma 1. For any $f \in H$ and $a \in A,\|f a\|=|[f] a|$.

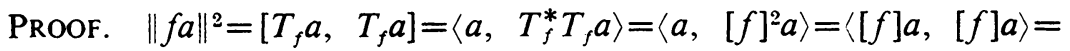
$|[f] a|^{2}$.

CoROLlaRY 1. $\|f\|_{\infty}=|[f]|_{\infty}=\left\|L_{[f]}\right\|=\left\|T_{f}\right\|=\left\|L_{f}\right\|$.

For any $f \neq 0$ in $H$, let $\sum \lambda_{n} e_{n}$ be the spectral representation of [ $\left.f\right]$ [7, Theorem 2.5]. We shall denote the countable (possibly finite) set $\left\{e_{n}\right\}$ by $E_{[f]}$ and refer to it as the spectral family of $[f]$. Any projection base $\left\{e_{\omega}: \omega \in \Omega\right\}$ containing every $e_{n} \in E_{[f]}$ will be called a projection base associated with [f]. In [1, Lemma 1] it is shown that if $\sum \lambda_{n} e_{n}$ is the spectral representation of $[f]$, then the operator $W_{f} \in R(A H)$ defined for any $x \in A$ by $W_{f} x=\sum \lambda_{n}^{-1} f e_{n} x$ is a partial isometry with $f=W_{f}[f]$ and $[f]=$ $W_{f}^{*} f$. We shall refer to $W_{f}$ as the partial isometry associated with $f$.

The proofs of our next two lemmas make use of the fact that for any $S \in R(A)$ and $1 \leqq p \leqq \infty$ we have $|S a|_{p} \leqq\|S\||a|_{p}$, where $\|S\|$ denotes the norm of $S$ as an operator on $(A,|\cdot|)$ [7, Proposition 3.19]. Lemma 2 gives a similar result for $T \in R(H A)$.

LEMMA 2. If $T \in R(H A)$, then $|T f|_{n} \leqq\|T\|\|f\|_{p}(1 \leqq p \leqq \infty)$.

Proof. $|T f|_{p}=\left|T W_{f}[f]\right|_{p} \leqq\left\|T W_{f}\right\||[f]|_{p} \leqq\|T\|\left\|W_{f}\right\|\|f\|_{p} \leqq\|T\|\|f\|_{p}$.

LEMMA 3. If $a \in A$ and $T \in R(H A)$, then there exists $g \in H$ such that $L_{a} T=L_{g}$; moreover, $\|g\|_{p} \leqq\|T\||a|_{n}(1 \leqq p \leqq \infty)$, where $\|T\|$ is the norm of $T$ as a transformation from $(H,\|\cdot\|)$ to $(A,|\cdot|)$.

Proof. The first part of the lemma is Lemma 7 of [1]. We shall show that $\|g\|_{p} \leqq\|T\||a|_{p}$, noting that this result is obvious for $p=\infty$, in view of Corollary 1. Assume now that $1 \leqq p<\infty$ and that $\sum \lambda_{n} e_{n}$ is the spectral representation of $[g] \neq 0$. As shown above, we have $T_{g}=L_{g}^{*}=$ $T^{*} L_{a}^{*}=T^{*} L_{a^{*}}$, so that $T_{g}[g]=g[g]=T^{*} a^{*}[g]$, and therefore

$$
W_{g}^{*} T^{*} a^{*}[g]=W_{g}^{*} g[g]=[g]^{2}=\sum \lambda_{n}^{2} e_{n} .
$$

It follows that $W_{g}^{*} T^{*} a^{*}[g] e_{n}=\lambda_{n}^{2} e_{n}=\lambda_{n} W_{g}^{*} T^{*} a^{*} e_{n}$, and we conclude that $W_{g}^{*} T^{*} a^{*} e_{n}=\lambda_{n} e_{n}=e_{n}\left(W_{g}^{*} T^{*} a^{*}\right)^{*}$. Now for any $b \in A$, let $P_{k} b=\sum_{n=1}^{k} e_{n} b$. 
$P_{k}$ is the orthogonal projection onto the right ideal $\sum_{n=1}^{k} e_{n} A$ in $A$, and $P_{k} \in R(A)$. We have

$$
P_{k}\left(W_{g}^{*} T^{*} a^{*}\right)^{*}=\sum_{n=1}^{k} e_{n}\left(W_{g}^{*} T^{*} a^{*}\right)^{*}=\sum_{n=1}^{k} \lambda_{n} e_{n} .
$$

Thus, for each $k$,

$$
\begin{aligned}
\sum_{n=1}^{k} \lambda_{n}^{p}\left|e_{n}\right|^{2} & =\left|P_{k}\left(W_{g}^{*} T^{*} a^{*}\right)^{*}\right|_{p}^{p} \leqq\left\|P_{k}\right\|^{p}\left|W_{g}^{*} T^{*} a^{*}\right|_{p}^{p} \\
& \leqq\left\|W_{g}^{*} T^{*}\right\|^{p}\left|a^{*}\right|_{p}^{p} \leqq\left\|W_{g}^{*}\right\|^{p}\left\|T^{*}\right\|^{p}|a|_{p}^{p} \leqq\|T\|^{p}|a|_{p}^{p}
\end{aligned}
$$

(We have used the fact that $|a|_{p}=\left|a^{*}\right|_{p}[7$, Corollary 3.16].) Therefore, $|[g]|_{p}^{p} \leqq\|T\|^{p}|a|_{p}^{p}$, or $\|g\|_{p} \leqq\|T\||a|_{p}$.

3. Proof of Theorem 1. The first statement of the theorem is evident from the corresponding statements about $A_{p}$ [7, Corollary 3.12]. To show that $H_{p}(1 \leqq p \leqq \infty)$ is a linear space we verify that $\|\cdot\|_{p}$ is a linear space norm, a fact which is obvious for $p=\infty$, by Corollary 1 . To establish subadditivity for $1 \leqq p<\infty$, let $f$ and $g$ be any elements of $H_{p}$, and let $W$ be the partial isometry associated with $f+g$. We then have

$$
\begin{aligned}
\|f+g\|_{n} & =\left|W^{*}(f+g)\right|_{p}=\left|W^{*} f+W^{*} g\right|_{p} \\
& =\left|W^{*} W_{f}[f]+W^{*} W_{g}[g]\right|_{p} \leqq\left|W^{*} W_{f}[f]\right|_{p}+\left|W^{*} W_{g}[g]\right|_{p} \\
& \leqq\left\|W^{*} W_{f}\right\||[f]|_{p}+\left\|W^{*} W_{g}\right\||[g]|_{p} \leqq\|f\|_{p}+\|g\|_{p} .
\end{aligned}
$$

We have again used Proposition 3.19 of [7], as well as the triangle inequality for $|\cdot|_{p}[7$, Proposition 3.23]. The remaining properties of a linear space norm are readily verified. Now for any $f \in H, a \in A$, let $W$ be the partial isometry associated with $f a$. We have $\|f a\|_{p}=|[f a]|_{p}=\left|W^{*} f a\right|_{p} \leqq$ $\left|W^{*} f\right|_{p}|a|_{\infty}$ by [7, Corollary 3.20]. Since $|a|_{\infty} \leqq|a|$ [7, Lemma 3.9],

$$
\left|W^{*} f\right|_{p}|a|_{\infty} \leqq\left\|W^{*}\right\|\|f\|_{p}|a| \leqq\|f\|_{p}|a| .
$$

Thus $H_{p}$ is a right normed $A$-module. The final statement of the theorem follows from corresponding results in $A$ [7, Remark 3.5], along with [1, Lemma 2]: for any $f \in H$,

and

$$
\|f\|_{2}^{2}=|[f]|_{2}^{2}=|[f]|^{2}=\operatorname{tr}[f]^{2}=\operatorname{tr}(f, f)=[f, f]=\|f\|^{2},
$$

$$
\|f\|_{1}=|[f]|_{1}=\tau([f])=\pi(f) .
$$

We remark that the completeness of $\left(H_{p},\|\cdot\|_{p}\right)$ for $1 \leqq p<2$ can be established by the method of [3, p. 265] as adapted in [1]; however we omit this proof since it is rendered unnecessary by Theorem 2 . 
4. Proof of Theorem 2. The case $p=1$ is Theorem 2 of [1]. For $1<p \leqq 2$ we observe that $2 \leqq q<\infty$. For any $g \in H_{q}(=H)$ and any $f \in H_{p}$, let $\phi_{f}(g)=[g, f]=\operatorname{tr}(f, g)$. Clearly, $\phi_{f}$ is a linear functional on $H_{q}$ and the mapping $f \rightarrow \phi_{f}$ is conjugate-linear. We shall show first that $\phi_{f}$ is bounded and that $\left\|\phi_{f}\right\| \leqq\|f\|_{p}$. If $E=\left\{e_{\omega}: \omega \in \Omega\right\}$ is any projection base for $A$, we have

$$
\begin{aligned}
\left|\phi_{f}(g)\right| & =|\operatorname{tr}(f, g)|=|\operatorname{tr}(g, f)|=\left|\sum\left\langle(g, f) e_{\omega}, e_{\omega}\right\rangle\right| \leqq \sum\left|\left\langle\left(g, f e_{\omega}\right), e_{\omega}\right\rangle\right| \\
& =\sum\left|\left[f e_{\omega}, g e_{\omega}\right]\right| \leqq \sum\left\|f e_{\omega}\right\|\left\|g e_{\omega}\right\|=\sum\left|[f] e_{\omega}\right|\left|[g] e_{\omega}\right|,
\end{aligned}
$$

by Lemma 1. If $E$ is now taken to be a projection base associated with [ $f$ ], we conclude as in the proof of [7, Lemma 3.25] that this last sum does not exceed $|[f]|_{p}|[g]|_{q}=\|f\|_{p}\|g\|_{q}$.

To show that $\|f\|_{p} \leqq\left\|\phi_{f}\right\|$, we consider the linear functional $\theta_{[f]}$ defined on $A_{q}$ by $\theta_{[f]}(a)=\operatorname{tr} a[f]$. From [7, Proposition 3.26], we have $\left\|\theta_{[f]}\right\|=$ $|[f]|_{p}=\|f\|_{p}$; hence it suffices to show that $\left\|\theta_{[f]}\right\| \leqq\left\|\phi_{f}\right\|$. Let $a$ be any element of $A_{q}$. Then

$$
\begin{aligned}
\left|\theta_{[f]}(a)\right| & =|\operatorname{tr} a[f]|=\left|\operatorname{tr} a W_{f}^{*} f\right|=\left|\operatorname{tr} L_{g} f\right| \\
& =|\operatorname{tr}(g, f)|=|\operatorname{tr}(f, g)|=\left|\phi_{f}(g)\right|,
\end{aligned}
$$

where, by Lemma 3, $g \in H$ is such that $L_{a} W_{f}^{*}=L_{g}$, with $\|g\|_{q} \leqq|a|_{q}$. Using this last inequality we conclude that $\left\|\theta_{[f]}\right\| \leqq\left\|\phi_{f}\right\|$.

We must show, finally, that the mapping $f \rightarrow \phi_{f}$ is onto the dual of $H_{q}$. Let $\phi$ be any bounded linear functional on $H_{q}$. For each $g \in H_{q}(=H)$, $|\phi(g)| \leqq\|\phi\|\|g\|_{q} \leqq\|\phi\|\|g\|$, since $q \geqq 2$.Thus $\phi$ is bounded on $(H,\|\cdot\|)$ and there exists $f \in H$ such that $\phi(g)=[g, f]$. We need only show that $f \in H_{p}$. Let $\sum \lambda_{n} e_{n}$ be the spectral representation of $[f]$, and let

$$
v_{k}=\sum_{n=1}^{k} \lambda_{n}^{p-1} e_{n} \in A_{q} ;
$$

then

$$
\left|v_{k}\right|_{q}=\left(\sum_{n=1}^{k} \lambda_{n}^{p q-a}\left|e_{n}\right|^{2}\right)^{1 / a}=\left(\sum_{n=1}^{k} \lambda_{n}^{p}\left|e_{n}\right|^{2}\right)^{1 / q} .
$$

Using Lemma 3, we take $g_{k} \in H$ such that $L_{g_{k}}=L_{v_{k}} W_{f}^{*}$, where $\left\|g_{k}\right\|_{q} \leqq\left|v_{k}\right|_{q}$. Then for each $k$,

$$
\begin{aligned}
\sum_{n=1}^{k} \lambda_{n}^{p}\left|e_{n}\right|^{2} & =\left|\operatorname{tr} \sum_{n=1}^{k} \lambda_{n}^{p} e_{n}\right|=\left|\operatorname{tr}\left(\sum_{n=1}^{k} \lambda_{n}^{p-1} e_{n}\right)[f]\right|=\left|\operatorname{tr} v_{k}[f]\right| \\
& =\left|\operatorname{tr} v_{k} W_{f}^{*} f\right|=\left|\operatorname{tr}\left(g_{k}, f\right)\right|=\left|\left[g_{k}, f\right]\right|=\left|\phi\left(g_{k}\right)\right| \\
& \leqq\|\phi\|\left\|g_{k}\right\|_{q} \leqq\|\phi\|\left|v_{k}\right|_{q}=\|\phi\|\left(\sum_{n=1}^{k} \lambda_{n}^{p}\left|e_{n}\right|^{2}\right)^{1 / q}
\end{aligned}
$$


Thus $\left(\sum_{n=1}^{k} \hat{\lambda}_{n}^{p}\left|e_{n}\right|^{2}\right)^{1 / p} \leqq\|\phi\|$ for each $k$, and consequently $\|f\|_{p} \leqq\|\phi\|<\infty$.

5. Conditions for distinctness of the $p$-classes. Suppose $1 \leqq p<p^{\prime} \leqq 2$. In the $H^{*}$-algebra $A$, for $A_{p}$ to be a proper subset of $A_{p^{\prime}}$ it is necessary and sufficient that $A$ be infinite-dimensional [7, Proposition 3.14]. We shall give a condition for the corresponding relationship to hold in the case of $H_{p}$ and $H_{p^{\prime}}$.

An element of $H$ will be called primitive if it is of the form $f e \neq 0$, where $e$ is a primitive projection in $A$; if $\|f e\|=|e|, f e$ will be called a normal primitive element. (Note that the primitive projection $e$ is uniquely determined for $f e$, since if $f e=g p$, where $p$ is a primitive projection in $A$, then $e(f, f) e=p(g, g) p=\alpha e=\beta p \neq 0$; hence $e=p$.) A pair of primitive elements $f_{1} e_{1}$ and $f_{2} e_{2}$ will be called doubly orthogonal if $\left(f_{1} e_{1}, f_{2} e_{2}\right)=$ 0 and $\left\langle e_{1}, e_{2}\right\rangle=0$.

Proposition 1. For $1 \leqq p<p^{\prime} \leqq 2, H_{p}$ is a proper subset of $H_{p^{\prime}}$ if and only if $H$ contains an infinite set of pairuise doubly orthogonal primitive elements.

Proof. We note first that there exist nonempty sets of pairwise doubly orthogonal primitive elements in $H$, since for any $f \in H$ there is a primitive projection $e$ such that $f e \neq 0$ (Lemma 1). Now suppose that every maximal set of such elements is finite, and let $\left\{f_{1} e_{1}, \cdots, f_{k} e_{k}\right\}$ be a maximal set. We may assume that the $f_{n} e_{n}$ are normal. We have $\left[f_{n} e_{n}\right]^{2}=$ $\left(f_{n} e_{n}, f_{n} e_{n}\right)=e_{n}\left[f_{n} e_{n}\right]^{2} e_{n}=\alpha_{n}^{2} e_{n}$ for positive $\alpha_{n}$, since $\left[f_{n} e_{n}\right]^{2}$ is a positive element of $A$. Hence $\left[f_{n} e_{n}\right]=\alpha_{n} e_{n}$, and from $\alpha_{n}\left|e_{n}\right|=\left|\left[f_{n} e_{n}\right]\right|=\left\|f_{n} e_{n}\right\|=\left|e_{n}\right|$ we conclude that $\alpha_{n}=1$. Thus for any $a \in H,\left\|f_{n} e_{n} a\right\|=\left|\left[f_{n} e_{n}\right] e_{n} a\right|=\left|e_{n} a\right|$, and hence $f_{n} e_{n} A$ is a closed submodule of $A$ isomorphic to the closed right ideal $e_{n} A$. Let $M=\sum_{n=1}^{k} f_{n} e_{n} A$. Then $H=M \ominus M^{p}$, where $M^{p}=\{f \in H$ : $(f, g)=0$ for all $g \in M\}$ [4, corollary to Lemma 3]. Clearly, every element of $M$ belongs to the trace class $\tau H$. We shall show that the same is true for elements of $M^{p}$. Let $\left\{e_{\omega}:(1) \in \Omega\right\}$ be a projection base for $A$ containing $\left\{e_{1}, \cdots, e_{k}\right\}$. For any $f \in M^{p}$ and any $e_{\alpha} \neq e_{n}(n=1, \cdots, k), f e_{\alpha}=0$ or else $f e_{\alpha}$ would be a primitive element doubly orthogonal to each $f_{n} e_{n}$ $(n=1, \cdots, k)$, contradicting maximality. Thus $[f] e_{x}=0$, by Lemma 1 , and we have $[f]=\sum[f] e_{\omega}=\sum_{n=1}^{k}[f] e_{n}$; hence $[f] \in \tau A$ and $f \in \tau H$. Since $H$ is identical with its trace class, $H_{p}=H$ for $1 \leqq p \leqq 2$.

Suppose, to the contrary, that $H$ contains an infinite set $\left\{f_{n} e_{n}: n \in N\right\}$ of pairwise doubly orthogonal (normal) primitive elements. For $1 \leqq p<$ $p^{\prime} \leqq 2$, choose $r$ with $p<r<p^{\prime}$, and consider the series $\sum n^{-1 / r}\left|e_{n}\right|^{-2 / p^{\prime}} f_{n} e_{n}$. The terms of this series are mutually orthogonal in $(H,\|\cdot\|)$; and, recalling from above that $\left[f_{n} e_{n}\right]=e_{n}$, we easily show that the squares of their norms have a finite sum. Thus there exists $f \in H$ such that $f=\sum n^{-1 / r}\left|e_{n}\right|^{-2 / p^{\prime}} f_{n} e_{n}$. 
Now by the continuity of $(\cdot, \cdot)$ on $H \times H$, we have $[f]^{2}=(f, f)=$ $\sum n^{-2 / r}\left|e_{n}\right|^{-4 / p^{\prime}}\left(f_{n} e_{n}, f_{n} e_{n}\right)=\sum n^{-2 / r}\left|e_{n}\right|^{-4 / p^{\prime}} e_{n} ;$ and therefore $[f]=$ $\sum n^{-1 / r}\left|e_{n}\right|^{-2 / p^{\prime}} e_{n}$. It is now a simple matter to show, just as in [7, Proposition 3.14], that $f \in H_{p^{\prime}}$, but $f \notin H_{p}$.

We close by remarking that for the condition of Proposition 1 to hold, $A$ must necessarily be infinite-dimensional, as is evident. This is not sufficient, however, as is shown by the Hilbert $A$-module $e A$, where $e$ is a primitive projection and $A$ is topologically simple (the latter condition assuring that the module is faithful). However, by means of Theorem 6 of [4], along with the accompanying examples, it is readily possible to provide instances of Hilbert $A$-modules possessing the property of Proposition 1 .

\section{REFERENCES}

1. G. R. Giellis, Trace-class for a Hilbert module, Proc. Amer. Math. Soc. 29 (1971), 63-68. MR 43 \#2523.

2. E. Hewitt and K. A. Ross, Abstract harmonic analysis. Vol. II: Structure and analysis for compact groups analysis on locally compact Abelian groups, Die Grundlehren der math. Wissenschaften, Band 152, Springer-Verlag, New York and Berlin, 1970. MR 41 \#7378.

3. C. A. McCarthy, $c_{n}$, Israel J. Math. 5 (1967), 249-271. MR 37 \#735.

4. P. P. Saworotnow, A generalized Hilbert space, Duke Math. J. 35 (1968), 191-197. MR 37 \#3333.

5. - Trace-class and centralizers of an $H^{*}$-algebra, Proc. Amer. Math. Soc. 26 (1970), 101-104. MR 42 \#2305.

6. P. P. Saworotnow and J. C. Friedell, Trace-class for an arbitrary $H^{*}$-algebra, Proc. Amer. Math. Soc. 26 (1970), 95-100. MR 42 \#2304.

7. J. F. Smith, The p-classes of an $H^{*}$-algebra, Pacific J. Math. (to appear).

Department of Mathematics, Le Moyne College, Syracuse, New York 13214 